\title{
Nefrolitotomía percutánea ambulatoria: serie de casos y revisión de la literatura
}

\section{Percutaneous Nephrolithotomy Ambulatory: Case Series and Literature Review}

\author{
Carlos Eduardo Hernández García ${ }^{1}$ Diana Chaparro ${ }^{2}$ Gustavo Ramos ${ }^{1}$ José de la Hoz ${ }^{3}$ \\ ${ }^{1}$ Miembro de la Sociedad Colombiana de Urología, Clínica Los \\ Nogales, Bogotá D.C. Colombia \\ ${ }^{2}$ Clínica La Colina, Bogotá D.C., Colombia \\ Address for correspondence Dr. Carlos Eduardo Hernández García, \\ MD, Clínica Los Nogales, Calle 95 № 23-61, Clínica Los Nogales. \\ Bogotá D.C., Colombia (e-mail: caeduardoher@gmail.com).
}

${ }^{3}$ Colsubsidio, Bogotá D.C., Colombia

Urol Colomb 2018;27:260-265.

\section{Resúmenes \\ Palabras Clave \\ - nefrolitotomía percutánea \\ - readmisión del paciente \\ - dolor posoperatorio \\ - atención ambulatoria \\ - complicaciones posoperatorias \\ - tiempo de internación \\ - resultado del tratamiento \\ - tempo operativo}

\section{Abstract}

Objetivo Nuestro objetivo es dar a conocer nuestra experiencia con 20 pacientes con cálculos renales llevados a nefrolitotomía percutánea ambulatoria, basados en criterios estrictos preoperatorios e intraoperatorios; fortaleciendo las ventajas de nuestra técnica como los son el egreso temprano, bajos requerimientos de analgésicos postoperatorios, alta tasa libre de cálculos y una baja frecuencia de complicaciones. Métodos Se realiza una investigación en la literatura en las bases de búsqueda. Mediante la aplicación de criterios prequirúrgicos, intraoperatorios y postoperatorios se realiza la selección de 20 pacientes para ser llevados a la realización de nefrolitotomía percutánea entre noviembre de 2015 y mayo de 2016.

Resultados Dentro de las características intraoperatorias se destacan el tiempo quirúrgico, el cual fue de 70 minutos y no se presentaron sangrados considerables que requirieran de transfusiones. No se presentaron complicaciones intraquirúrgicas. El egreso fue durante las 8 primeras horas del postoperatorio. Se realizó seguimiento de dolor; evidenciando a las 24 horas que el $100 \%$ de los pacientes presentaban dolor $=\mid<4 / 10$. El control imagenológico se realizó con uroTAC observando una tasa libre de cálculos menores de $2 \mathrm{~mm}$ del $90 \%$ y menores de $4 \mathrm{~mm}$ en el $95 \%$. Dentro de las complicaciones presentadas se encontraron 2 reingresos que se manejaron ambulatoriamente sin complicaciones. Conclusiones La NLP ambulatoria es una opción de tratamiento seguro, con excelente tasa de éxito. Herramientas prequirúrgicas como los nomogramas permiten evaluar las características del cálculo con el fin de ofrecer mejores tasas de éxito, procedimientos con menor tasa de complicaciones y seguros.

Objective Our goal is to present our experience with 20 patients with renal calculi who underwent ambulatory percutaneous nephrolithotomy, based on strict preoperative and intraoperative criteria; Strengthening the advantages of our technique such as early discharge, low requirements of postoperative analgesics, high rate of free calculations and a low frequency of complications. received

November 27, 2016

accepted

February 8, 2018

published online

July 10,2018
DOI https://doi.org/

10.1055/s-0038-1645843.

ISSN 0120-789X.

eISSN 2027-0119.
Copyright $\odot 2018$, Sociedad Colombiana License terms de Urología. Publicado por Thieme Revinter Publicações Ltda., Rio de Janeiro, Brazil. Todos los derechos reservados.
() (1) $\ominus \circledast$ 


\section{Keywords}

- nephrostomy

- percutaneous

- patient readmission

- pain

- postoperative

- ambulatory care

- postoperative complications

- length of stay

- treatment outcome

- operative time
Methods We performed an investigation in the literature in the search bases. Through the application of preoperative, intraoperative and postoperative criteria, we selected 20 patients to be submitted to percutaneous nephrolithotomy between November 2015 and May 2016.

Results Regarding the intraoperative characteristics, the surgical time can be emphasized, which was 70 minutes and there were no significant bleeding that required transfusions. There were no intraoperative complications. The discharge was during the first 8 hours postoperatively. Pain follow-up was performed; evidencing at 24 hours that $100 \%$ of patients presented pain $=\mid<4 / 10$. The control was performed with uroTAC observing a free rate of stones less than $2 \mathrm{~mm}$ of $90 \%$ and less than $4 \mathrm{~mm}$ in 95\%. Among the complications presented were 2 readmissions that were managed ambulatorily without complications.

Conclusions Ambulatory NLP is a safe treatment option, with an excellent success rate. Preoperative tools such as nomograms allow the evaluation of the characteristics of the stone, with the porpouse to offer better success rates, procedures with lower complication rates and insurance.

\section{Introducción}

La nefrolitotomía percutánea (PCNL), es un procedimiento mínimamente invasivo, considerado como el tratamiento de elección para el manejo de los cálculos renales de gran tamaño o de formación compleja. Esa técnica fue introducida en la urología desde hace más de 30 años. Inicialmente fue Goodwin en 1955 quien realizó el primer abordaje percutáneo en un riñón. ${ }^{1}$ Posteriormente en 1976 Fernstórm y Johansson, efectuaron la primera extracción de un cálculo renal por vía percutánea ${ }^{2}$; permitiendo así el inicio y desarrollo de una nueva técnica mínimamente invasiva para el manejo de la litiasis renal.

Aquella técnica, si bien presenta menor morbilidad que la cirugía abierta, no es un procedimiento libre de complicaciones, por lo cual se han realizado una seria de modificaciones de la técnica inicial.

Adicionalmente, varios autores han propuesto diferentes sistemas de escalas que sugieren previamente el grado de complejidad del caso y la tasa de éxito posterior a la nefrolitotomía percutánea. Se encuentran actualmente múltiples escalas, algunas han sido reconocidas como la de Guy, el sistema de puntaje de nefrolitometría S.T.O.N.E y el nomograma de la Oficina de Investigación clínica de la Sociedad de Endourología (CROES). ${ }^{3}$ Los cuales proporcionan una herramienta útil para el urólogo y su paciente en el momento de discutir las mejores posibilidades terapéuticas.

Los parámetros del sistema S.T.O.N.E se encuentran basados en criterios estrictos de los hallazgos imagenológicos del TAC realizado durante la valoración inicial del paciente, teniendo en cuenta el tamaño del cálculo (S), topografía o localización del lito/ longitud pielcalculo $(\mathrm{T})$, obstrucción/grado de hidronefrosis y/o la presencia de stent ureteral $(0)$, el número de cálculos y cálices comprometidos(N) y la evaluación de la densidad del cálculo de acuerdo a unidades Hounsfield (E). Esos criterios establecen una puntuación final que se considera favorable entre 5 y 13 para lograr un éxito posoperatorio cercano al $100 \%$, definido como un estado libre de cálculo con fragmentos residuales menores de $4 \mathrm{~mm}^{4}$

Las medidas del cálculo se toman en $\mathrm{mm}^{2}$ teniendo en cuenta el eje mayor en el plano transverso y longitudinal, lo que permite asignar un puntaje de acuerdo a la medida calculada ( - Tabla 1). La distancia piel cálculo se expresa en $\mathrm{mm}$, tomando como punto limítrofe para la categorización $100 \mathrm{~mm}$ es decir $(</=$ de $100 \mathrm{~mm}$ : 1 punto $o>$ de 100mm: 2 puntos). Al grado de hidronefrosis se le asigna una puntuación de acuerdo a la severidad; leve, moderada o severa. El cuarto componente que hace referencia al compromiso calicial se puntúa de acuerdo al número de sistemas comprometidos. Y la densidad tiene un punto de corte en $</=950$ UHF y $>950$ UHF. $^{5}$

Por otra parte Thomas y col. propusieron el sistema de puntaje de Guy para calcular el grado de complejidad de la nefrolitotomía percutánea y predecir la tasa libre de cálculos posterior a la PCNL, basado en los hallazgos de los estudios imagenológicos usando radiografías de riñones, uréteres y vejiga, pielografía intravenosa oTAC sin contraste además de incluir algunas variables propias del paciente. ${ }^{6}$

Tradicionalmente la PCNL es un procedimiento que requiere en el posoperatorio inmediato, la observación del paciente durante un tiempo variable dentro de la institución, sin embargo, las múltiples modificaciones que se han logrado han permitido disminuir ese periodo; el uso de nefrostomías de menor calibre, el uso de catéteres ureterales y la creación de las escalas que permiten seleccionar aquellos pacientes cuyos casos son aptos para realizar un procedimiento ambulatorio, han permitido que la PCNL continúe evolucionando, siendo cada vez más segura y con alta efectividad.

Nuestro objetivo es dar a conocer nuestra experiencia con 20 pacientes con cálculos renales llevados a nefrolitotomía percutánea ambulatoria, basados en criterios estrictos 
Tabla 1 Variables del sistema de nefrolitometría S.T.O.N.E score

\begin{tabular}{|c|c|c|c|c|c|}
\hline Variable & 1 & 2 & 3 & 4 & \\
\hline Tamaño del cálculo $\left(\mathrm{mm}^{2}\right)$ & $0-399$ & $400-799$ & \multicolumn{2}{|c|}{$800-1599$} & $\geq 1600$ \\
\hline Longitud del tracto $(\mathrm{mm})$ & $\leq 100$ & $>100$ & & \\
\hline Obstrucción & ninguna & severa & & & \\
\hline Calices (n) & $1-2$ & 3 & \multicolumn{2}{|c|}{ Staghorn Stone } & \\
\hline \multirow[t]{3}{*}{ Essence (HU) } & $\leq 950$ & $>950$ & & & \\
\hline & \multicolumn{4}{|c|}{ Tamaño del cálculo Residual } & \\
\hline & $\leq 2 \mathrm{~mm}$ & 3-4 mm & $>4 \mathrm{~mm}$ & Total & $\mathrm{P}$ valor \\
\hline \multicolumn{5}{|l|}{ Tamaño del cálculo $\left(\mathrm{mm}^{2}\right)$} & 0.019 \\
\hline $0-399$ & 20 & 7 & 3 & 30 & \\
\hline $400-799$ & 19 & 4 & 5 & 28 & \\
\hline $800-1599$ & 19 & 5 & 8 & 32 & \\
\hline$\geq 1600$ & 9 & 10 & 13 & 32 & \\
\hline \multicolumn{5}{|l|}{ Longitud del tracto $(\mathrm{mm})$} & 0.071 \\
\hline$\leq 100$ & 22 & 7 & 3 & 32 & \\
\hline$>100$ & 45 & 19 & 26 & 90 & \\
\hline \multicolumn{5}{|l|}{ Obstrucción } & 0.318 \\
\hline No/mild hydronephrosis & 34 & 13 & 10 & 57 & \\
\hline Moderate/severe hydronephrosis & 33 & 13 & 19 & 65 & \\
\hline \multicolumn{5}{|l|}{ Número de cálices con cálculo } & $<0.001$ \\
\hline 1 & 21 & 1 & 1 & 23 & \\
\hline $2-3$ & 18 & 9 & 3 & 30 & \\
\hline$>3$ and stahorn & 28 & 16 & 25 & 69 & \\
\hline \multicolumn{5}{|l|}{ Essence (HU attenuation) } & 0.649 \\
\hline$\leq 950$ & 47 & 20 & 19 & 86 & \\
\hline$>950$ & 20 & 6 & 10 & 36 & \\
\hline
\end{tabular}

preoperatorios e intraoperatorios; fortaleciendo las ventajas de nuestra técnica como los son el egreso temprano, bajos requerimientos de analgésicos postoperatorios, alta tasa libre de cálculos y una baja frecuencia de complicaciones.

\section{Materiales y Métodos}

Se realiza una investigación en la literatura en la bases de búsqueda Pubmed, science direct, Scopus y Embase.

Palabras clave utilizadas: ambulatory percutaneous nephrolithotomy, rate of readmission, stone score, postoperative discomfort, postoperative complications.

Mediante la aplicación de criterios prequirúrgicos, intraoperatorios y postoperatorios, se realiza la selección de 20 pacientes para ser llevados a la realización de nefrolitotomía percutánea entre noviembre de 2015 y mayo de 2016.

Dentro de los criterios de inclusión prequirúrgicos se encuentran: criterios de la Sociedad Americana de Anestesiología (ASA) $<3$, e índice de masa corporal $<35 \mathrm{~kg} / \mathrm{m} 2$. Se excluyen pacientes con riñón único o trasplantado, enfermedad cardiaca activa, insuficiencia renal, así como antecedentes de IVU a repetición. Dentro de los criterios de inclusión intraoperatorios se encuentran: número de punciones menor a 3, requerir un único tracto, remoción completa del cálculo, sangrado escaso y la ausencia de perforación de la pelvis renal. Dentro de los criterios postoperatorios se encuentran la estabilidad hemodinámica y adecuado control del dolor. ${ }^{7,8}$

Adicionalmente se tienen en cuenta los parámetros pronósticos de éxito quirúrgico en nefrolitotomía percutánea clasificados por los criterios del S.T.O.N.E score, considerando como éxito quirúrgico la eliminación del cálculo con fragmentos residuales menores de $2 \mathrm{~mm}$. Se tuvieron en cuenta imágenes tomográficas prequirúrgicas y posoperatorias llevadas a cabo durante las 4 primeras semanas luego del procedimiento.

Todos los procedimientos fueron llevados a cabo en posición de decúbito dorsal, usando balón neumático para la dilatación del tracto. Para la litofragmentación se utilizó litoclast en la mayoría de los casos y en algunos láser.

Todos los pacientes fueron manejados con una derivación urinaria, ya fuera catéter doble J o nefrostomía. 
En todos los pacientes el egreso se realizó el mismo día de la realización del procedimiento, aproximadamente entre las primeras 8 horas del posoperatorio. Se realizó revaloración del paciente a las 24 horas de manera ambulatoria, efectuando un seguimiento analgésico mediante la escala análoga el dolor y se vigiló la aparición de signos asociados a la infección, sangrado o signos irritativos urinarios.

Para el análisis estadístico se utilizó el programa stata 12.0. Se realizó un análisis de cada una de las variables del cálculo y así mismo se evaluaron todas las variables en conjunto utilizadas en el score de S.T.O.N.E. Se utilizan pruebas estadísticas de chi cuadrado y valores de $p<0.05$ como estadísticamente significativos.

\section{Resultados}

Se incluyeron 20 pacientes, 12 hombres y 8 mujeres con promedio de edad de 49 años (-Tabla 2).

Dentro de las características intraoperatorias se destacan (-Tabla 3), el tiempo promedio de cirugía, el cual fue de 70 minutos, no se presentaron sangrados considerables que requirieran de transfusiones. Al final del procedimiento la mitad de los pacientes fueron manejados con nefrostomía 24Fr en su mayoría y la otra mitad con catéter doble j.

No se presentaron complicaciones intraquirúrgicas. En el caso de los pacientes con nefrostomía se retiró alrededor del 5to día POP y el catéter doble J alrededor de las 3 semanas luego de su autorización administrativa.

El egreso se dio durante las 8 primeras horas POP. El manejo analgésico ambulatorio incluía, antiespasmódicos, AINES y ocasionalmente tramadol. En ninguno de los casos se utilizó morfina o hidromorfona.

Se realizó seguimiento con escala análoga de dolor de 0 a 10 . A las 6 horas POP la mayoría de pacientes presentaron un dolor de 5/10. A las 12 horas, la escala análoga mostraba que un $80 \%$ de los pacientes presentaban dolor de $3-4 / 10$, finalmente a las

Tabla 2 Características de base de la población y cálculos

\begin{tabular}{|l|l|}
\hline $\begin{array}{l}\text { Características del } \\
\text { Paciente y Lito }\end{array}$ & $\begin{array}{l}\text { No (\%)/Promedio } \\
\text { (Rango) }\end{array}$ \\
\hline Total población & 20 pacientes \\
\hline Edad Promedio (años) & $49,9(35-66)$ \\
\hline Sexo: Femenino:, masculino & $8(40 \%), 12(60 \%)$ \\
\hline IMC (Kg/m ${ }^{2}$ ) & $29,4(25-34)$ \\
\hline ASA I, II & $6(30 \%), 14(70 \%)$ \\
\hline Lateralidad: Derecho, Izquierdo & $8(40 \%), 12(60 \%)$ \\
\hline Factores del Lito & $788(90-1600)$ \\
\hline Tamaño promedio (mm $\left.{ }^{2}\right)$ & $103(80-150)$ \\
\hline $\begin{array}{l}\text { Distancia Piel Cálculo } \\
\text { promedio (mm) }\end{array}$ & $11(55 \%), 9(45 \%)$ \\
\hline Grado Hidronefrosis 0-I, II-III. & $1009(600-1300)$ \\
\hline Densidad UH & $17(85 \%), 3(15 \%)$ \\
\hline $\begin{array}{l}\text { Numero de cálices } \\
\text { comprometidos I, II, III. }\end{array}$ \\
\hline
\end{tabular}

Tabla 3 Características Intraoperatorias

\begin{tabular}{|l|l|}
\hline Variable intraoperatoria & No (\%) \\
\hline Características del Calculo & $10(50)$ \\
\hline Pielico & $4(20)$ \\
\hline Pielico + Calicial Inferior & $3(15)$ \\
\hline Calicial Inferior & $1(5)$ \\
\hline Calicial medio & $1(5)$ \\
\hline Calicial Superior & $1(5)$ \\
\hline Unión pielo ureteral & $14(70)$ \\
\hline Sitio de Punción & $5(25)$ \\
\hline Calicial Inferior & $1(5)$ \\
\hline Calicial medio & $17(85)$ \\
\hline Calicial Superior & $3(15)$ \\
\hline Litotripsia Intracorpórea & $10(50)$ \\
\hline Neumática & $10(50)$ \\
\hline Láser & \multicolumn{2}{|l}{} \\
\hline Derivación Utilizada & \\
\hline Nefrostomía & \\
\hline Catéter doble j & \\
\hline
\end{tabular}

24 horas, el $80 \%$ presentaban dolor de $2-3 / 10$ y el $100 \%$ dolor $=\mid<4 / 10$.

El control imagenológico se realizó durante las 4 primeras semanas POP con UroTAC observando una tasa libre de cálculos menores de $2 \mathrm{~mm}$ del $90 \%$ y menores de $4 \mathrm{~mm}$ en el $95 \%$.

En el análisis estadístico (ver - Tabla 4), la variable que más se correlaciona con éxito quirúrgico es el tamaño del cálculo, sin embargo solo el puntaje de STONE cuyo promedio fue de 8 , presento una asociación estadísticamente significativa con una $p<0,05$ en relación al éxito postoperatorio de cálculos menores a $2 \mathrm{~mm}$.

Dentro de las complicaciones presentadas se encontraron 2 reingresos: un paciente con cólico renoureteral y hematuria asociado a la presencia del catéter doble $\mathrm{J}$ y el segundo por IVU a los 8 días que se trató con antibioticoterapia ambulatoriamente sin complicaciones.

\section{Discusión}

La evolución que ha tenido la nefrolitomía percutánea ha llevado a una disminución en los tiempos quirúrgicos, así como en la estancia intrahospitalaria. La pronta incorporación a la vida diaria, además de los escasos requerimientos analgésicos y la baja probabilidad de requerir hemotransfusión son parámetros que requieren herramientas adecuadas de planificación quirúrgica. Es allí donde el uso de ciertos criterios claros imagenológicos permiten que ese procedimiento se pueda planificar, con una adecuada predicción de la complejidad quirúrgica a la que nos vamos a enfrentar, de manera que podamos incluso tener criterios que permitan realizar una NLP ambulatoria.

Beiko y col. realizaron una revisión retrospectiva de 50 casos de pacientes a quienes se les realizó PCNL 
Tabla 4 Asociación estadística de las Variables de Parámetros de S.T.O.N.E en el análisis de sus variables y puntaje total en relación al éxito quirúrgico (cálculos residuales menores a $2 \mathrm{~mm}$ )

\begin{tabular}{|c|c|c|c|c|c|}
\hline \multirow[b]{2}{*}{ Variable } & \multicolumn{4}{|c|}{ Tamaño del cálculo Residual } & \multirow[b]{2}{*}{$P$ valor } \\
\hline & $\leq 2 \mathrm{~mm}$ & $3-4 \mathrm{~mm}$ & $>4 \mathrm{~mm}$ & Total & \\
\hline $\mathrm{N}$ & 18 & 1. & 1 & 20 & \\
\hline \multicolumn{5}{|c|}{ Tamaño del cálculo $\left(\mathrm{mm}^{2}\right)$} & 0.058 \\
\hline $0-399$ & 3 & & & 3 & \\
\hline $400-799$ & 8 & 1 & & 9 & \\
\hline $800-1599$ & 7 & & & 7 & \\
\hline$\geq 1600$ & & & 1 & 1 & \\
\hline \multicolumn{5}{|c|}{ Longitud del tracto $(\mathrm{mm})$} & 0.58 \\
\hline$\leq 100$ & 11 & 1 & & 12 & \\
\hline$>100$ & 7 & & 1 & 8 & \\
\hline \multicolumn{5}{|l|}{ Hidronefrosis } & 0.18 \\
\hline $0-1$ & 11 & & & 11 & \\
\hline IIIIII & 7 & 1 & 1 & 9 & \\
\hline \multicolumn{5}{|c|}{ Número de cálices con cálculo } & 0.2 \\
\hline $1-2$ & 18 & 1 & 1 & 20 & \\
\hline \multicolumn{6}{|l|}{3} \\
\hline \multicolumn{6}{|l|}{ coraliforme } \\
\hline \multicolumn{5}{|l|}{ Densidad UH } & 0.7 \\
\hline$\leq 950$ & 7 & & & 7 & \\
\hline$>950$ & 11 & 1 & 1 & 13 & \\
\hline \multicolumn{5}{|c|}{ S.T.O.N.E score } & 0.005 \\
\hline $5-10$ & 18 & & & 18 & \\
\hline $10-13$ & & 1 & 1 & 2 & \\
\hline$>13$ & & & & & \\
\hline
\end{tabular}

ambulatoria en dos instituciones entre marzo de 2007 y diciembre de 2013, teniendo en cuenta datos pre, intra y postoperatorios además de información acerca de la dosis de narcóticos, tiempo de estadía hospitalaria, complicaciones postoperatorias $y$ el reingreso a urgencias, evidenciando que la PCNL ambulatoria es un procedimiento seguro con una tasa libre de cálculos que alcanza el $90 \%$ y una tasa de reingresos del $4 \%$, datos similares a los presentados en nuestra serie. ${ }^{9}$

Es bien conocido que cada vez se busca disminuir la invasión del paciente en el posoperatorio, por lo que se busca generar tractos más pequeños dando egreso con catéteres de nefrostomía de menor tamaño, catéter doble J, o simplemente sin ninguna derivación.

Autores como Walid Shahrour y Sero Andonian, realizan también trabajos en esa corriente. En su estudio, decidieron evaluar la seguridad y la viabilidad de llevar a cabo NLP en 10 pacientes a los que se les realizó dicho procedimiento con colocación de catéter ureteral, en quienes se tomó la decisión de dar de alta el mismo día del procedimiento, basado en criterios pre, intra y posoperatorios de cada paciente.

Durante su estudio evidenciaron, con respecto a los hallazgos intraoperatorios, un tiempo promedio quirúrgico y de fluoroscopia de 83,5 minutos, escaso sangrado, sin requerimientos de transfusión. Consecutivamente los pacientes duraron aproximadamente 240 minutos en recuperación y se administró $19.25 \mathrm{mg}$ de morfina, con posterior egreso hospitalario y el uso de analgésicos en casa en tan solo 3 pacientes. Con respecto a las complicaciones posoperatorias, se presentaron 2 casos, el primero con infección de vías urinarias por E.coli multiresistente que requirió administración de antibioticoterapia endovenosa, y el segundo con TVP que requirió anticoagulación con heparina de bajo peso molecular. Concluyendo así que para pacientes determinados por ciertos criterios, la PCNL ambulatoria es segura y viable. ${ }^{10}$

En el presente estudio también usamos criterios preoperatorios en relación al IMC, criterios de ASA, procedimiento sin complicaciones, así como los criterios de STONE. Todo ello permitió tener los resultados encontrados.

Clásicamente la PCNL ha requerido un manejo intrahospitalario para vigilar la aparición de síntomas asociados a dolor, sangrado y sepsis, sin embargo, en la búsqueda de disminuir ese tiempo dentro de la institución, se han implementado herramientas extensamente aceptadas, sencillas y fáciles de usar como la escala de S.T.O.N.E. que permite tener un mejor planeamiento preoperatorio, y así una mayor tasa libre de cálculos con una subsecuente mayor tasa de éxito.

\section{Conclusión}

La NLP ambulatoria es una opción de tratamiento seguro, con excelente tasa de éxito. Herramientas prequirúrgicas como los nomogramas permiten evaluar las características del cálculo con el fin de ofrecer mejores tasas de éxito, procedimientos con menor tasa de complicaciones y más seguros. Sin embargo, se necesita realizar estudios adicionales en esa área para obtener más datos que nos permitan realizar adelantos en la técnica y sus resultados. Actualmente estamos llevando a cabo un estudio prospectivo aleatorizado con balón neumático, que promete resultados en el progreso de nuestra investigación.

\section{Responsabilidades Éticas}

Protección de personas y animales.

Los autores declaran que para esta investigación no se han realizado experimentos en seres humanos ni en animales.

Confidencialidad de los datos.

Los autores declaran que han seguido los protocolos de su centro de trabajo sobre la publicación de datos de pacientes.

Derecho a la privacidad y consentimiento informado.

Los autores declaran que en este artículo no aparecen datos de pacientes.

Note

No hubo fuente de financiación. No hubo conflicto de intereses. 


\section{Agradecimientos}

Agradecemos de ante mano a la clínica Los Nogales por su apoyo logístico y técnico que nos brindaron a lo largo de la creación del presente artículo.

\section{Bibliografia}

1 Goodwin WE, Casey WC, Woolf W. Percutaneous trocar (needle) nephrostomy in hydronephrosis. J Am Med Assoc 1955;157(11): 891-894

2 Fernström I, Johansson B. Percutaneous pyelolithotomy. A new extraction technique. Scand J Urol Nephrol 1976;10(03):257-259

3 Tailly T, Razvi H. The S.T.O.N.E. nephrolithometry scoring system: How valid is it? Can Urol Assoc J 2015;9(5-6):196

4 Akhavein A, Henriksen C, Syed J, Bird VG. Prediction of single procedure success rate using S.T.O.N.E. nephrolithometry surgical classification system with strict criteria for surgical outcome. Urology 2015;85(01):69-73

5 Okhunov Z, Friedlander JI, George AK, y col . S.T.O.N.E. nephrolithometry: novel surgical classification system for kidney calculi. Urology 2013;81(06):1154-1159

6 Thomas K, Smith NC, Hegarty N, Glass JM. The Guy's stone scoregrading the complexity of percutaneous nephrolithotomy procedures. Urology 2011;78(02):277-281

7 Shahrour W, Andonian S. Ambulatory percutaneous nephrolithotomy: initial series. Urology 2010;76(06):1288-1292

8 Beiko D, Lee L. Outpatient tubeless percutaneous nephrolithotomy: the initial case series. Can Urol Assoc J 2010;4(04):E86-E90

9 Beiko D, Elkoushy MA, Kokorovic A, Roberts G, Robb S, Andonian S. Ambulatory percutaneous nephrolithotomy: what is the rate of readmission? J Endourol 2015;29(04):410-414

10 Shahrour W, Andonian S. Ambulatory percutaneous nephrolithotomy: initial series. Urology 2010;76(06):1288-1292 